\title{
Analysis of the effect of neutral flow on the waves in the solar photosphere
}

\author{
D. Petrović ${ }^{1,2}$, J. Vranjes $^{1}$, and S. Poedts ${ }^{1}$ \\ ${ }^{1}$ Center for Plasma Astrophysics, K.U.Leuven, Celestijnenlaan 200B, 3001 Leuven, Belgium \\ e-mail: Dragana.Petrovic@wis. kuleuven. be \\ 2 Institute of Physics, PO Box 57, 11001 Belgrade, Serbia
}

Received 6 June 2006 / Accepted 31 July 2006

ABSTRACT

\begin{abstract}
Context. The solar surface and photosphere are covered by a network of convective motions of a mainly neutral fluid. Such a neutral motion drags the tiny plasma population along, which results in drifts of the plasma species due to the magnetic field. These drifts can, in turn, excite and amplify plasma perturbations, which is the subject of the present work.

Aims. The behaviour of electromagnetic waves is discussed for a weakly ionized plasma with a neutral flow, in a magnetization regime in which an electron drift exists relative to the ions. This drift across the magnetic field is caused by the neutral flow.

Methods. Using a standard normal mode approach, the linear dynamics of small perturbations propagating obliquely to the equilibrium magnetic field lines is investigated. In the regime of strong perturbations, in which the convective derivatives in the electron and ion momentum equations are within the same order of magnitude as the time derivatives, a nonlinear analysis is performed by considering spatial scales at which the effects due to collisions can be neglected.

Results. A dispersion relation describing the coupled, drift-driven, and dispersive Alfvén modes is obtained for a strongly collisional plasma. The results are applied to the solar photosphere. Without electron drift due to the frequent collisions, the real part of the (kinetic) Alfvén wave frequency practically vanishes; i.e., the KAW is completely damped. It is shown that the KAW is much less damped in the presence of the electron drift. However, the kinetic Alfvén wave cannot be destabilized by this drift. The instability of the drift-driven mode (Farley-Buneman type) is shown to develop when the electron drift exceeds a certain threshold. At spatial scales far exceeding the mean free path of the particles, the non-linear effects result in a self-organization in the form of traveling double
\end{abstract} vortices.

Key words. waves - instabilities - Sun: photosphere

\section{Introduction}

The mainly neutral fluid in the solar surface and the photosphere is moving in a certain pattern due to convection. Moreover, the photosphere is permeated by a magnetic carpet. The matter in the photosphere is weakly ionized, and this neutral motion drags the tiny plasma population along. Due to the magnetic field, this results in drifts of the plasma species. These drifts can, in turn, excite and amplify plasma perturbations. In the present paper, we analyse the effect of the neutral flow on the waves and instabilities in the solar photosphere.

According to the dynamo theory, a conductor moving across magnetic field lines produces an electric field perpendicular both to this magnetic field and to the velocity vector when the following conditions (the "dynamo inequalities") are satisfied:

$\frac{\Omega_{\mathrm{e}}}{v_{\mathrm{e}}} \gg 1$

and

$1 \ll \frac{\Omega_{\mathrm{e}} \Omega_{\mathrm{i}}}{v_{\mathrm{e}} v_{\mathrm{i}}} \ll \infty$

Here, $v_{\mathrm{e}}$ and $v_{\mathrm{i}}$ denote the electron and ion collision frequencies, respectively, and $\Omega_{\mathrm{e}}$ and $\Omega_{\mathrm{i}}$ are the electron and ion cyclotron frequencies (Sen \& White 1972; Khodachenko \& Zaitsev 2002).
The physical meaning of the dynamo theory is that, in the initial magnetic field, the ions are less closely bound to the magnetic field lines than the electrons. As a result, a flow of neutral atoms can carry the ions along more easily than the electrons in such a system. Because of that, a flow of neutral atoms can induce a charge separation in a partially ionized inhomogeneous plasma. When this happens, a charge-separation electric field occurs, and this field reaches a limiting value in the ambipolar diffusion regime, i.e., when the electrons and the ions diffuse at the same rate.

In nature, such perpendicular electric fields exist, for example, in the Earth's ionosphere $(h \simeq 85-110 \mathrm{~km})$ and in the solar photosphere. Both systems contain regions with weakly ionized plasma where the dynamo inequalities (1) and (2) are satisfied. This means that the neutral flow in the photosphere can indeed produce these perpendicular electric fields, which, in combination with the magnetic fields, causes an $\boldsymbol{E} \times \boldsymbol{B}$-drift. Due to the different magnetization regime, described by the dynamo inequalities, a relative drift of the electrons to the ions will appear. In the present paper, we investigate and discuss the stability of electromagnetic waves in a weakly ionized, strongly collisional plasma with such an electron drift across the magnetic field.

In other words, we consider the parameter regime in which the electrons are strongly magnetized, i.e., $v_{\mathrm{e}} \ll \Omega_{\mathrm{e}}$, and the ion-collision frequency is lower but in the same range as the ion cyclotron frequency, i.e. $v_{\mathrm{i}} \lesssim \Omega_{\mathrm{i}}$. Notice that in this parameter 
range the above dynamo inequalities (1) and (2) are satisfied. Thus, a relative electron drift can be generated in this regime by a charge separation electric field (induced by the above-mentioned advection by neutral atoms) perpendicular to the magnetic field. Hereafter, we will refer to this relative drift of the electrons to the ions as the "electron drift".

In Sect. 2 the physical model and the governing equations are presented and discussed. In Sect. 3 small (linear) perturbation are considered in a normal mode analysis that yields the wave dispersion equation. In Sect. 4 some numerical solutions of this dispersion equation are obtained and discussed for typical solar photosphere plasma parameter values. Next, the nonlinear regime is analyzed in Sect. 5. Finally, some concluding remarks are given in Sect. 6.

\section{Model and basic equations}

We want to discuss the influence of the equilibrium electron drift perpendicular to the magnetic field lines. The dynamics of this system, in the regime mentioned above, is described by the electron continuity equation

$\frac{\partial n_{\mathrm{e}}}{\partial t}+\nabla\left(n_{\mathrm{e}} \boldsymbol{v}_{\mathrm{e}}\right)=0$

the equations of motions for both the electrons and the ions

$$
\frac{e}{m_{\mathrm{e}}}\left(\boldsymbol{E}+\boldsymbol{v}_{\mathrm{e}} \times \boldsymbol{B}\right)+v_{\mathrm{Te}}^{2} \frac{\nabla n_{\mathrm{e}}}{n_{\mathrm{e}}}+v_{\mathrm{en}}\left(\boldsymbol{v}_{\mathrm{e}}-\boldsymbol{v}_{\mathrm{n}}\right)+v_{\mathrm{ei}}\left(\boldsymbol{v}_{\mathrm{e}}-\boldsymbol{v}_{i}\right)=0,
$$

$\left(\frac{\partial}{\partial t}+\boldsymbol{v}_{\mathrm{i}} \nabla\right) \boldsymbol{v}_{\mathrm{i}}=-v_{\mathrm{Ti}}^{2} \frac{\nabla n_{\mathrm{i}}}{n_{\mathrm{i}}}+\frac{e}{m_{\mathrm{i}}}\left(\boldsymbol{E}+\boldsymbol{v}_{\mathrm{i}} \times \boldsymbol{B}\right)-v_{\mathrm{in}}\left(\boldsymbol{v}_{\mathrm{i}}-\boldsymbol{v}_{\mathrm{n}}\right)-\frac{\nabla \pi_{i}}{m_{\mathrm{i}} n_{\mathrm{i}}},(5)$

and the quasi-neutrality equation

$\nabla \cdot \boldsymbol{j}=0$

where the subscripts e and i denote the corresponding quantities for electrons and ions, respectively. Here $\boldsymbol{v}_{\mathrm{n}}$ is the equilibrium neutral velocity, which is the driving force for the instability discussed here. The thermal velocity is denoted by $v_{\mathrm{Te}(\mathrm{i})}$, the electron(ion)-neutral collision frequency by $v_{\mathrm{e}(\mathrm{i}) \mathrm{n}}$, the electronion collision frequency by $v_{\mathrm{ei}}$, the anisotropic stress tensor by $\pi_{\mathrm{i}}$, while all the other symbols retain their conventional meanings.

Notice that viscous effects are not included. As a matter of fact, these effects are negligible here since the effects of collisions with neutrals dominate for the considered set of parameters and for the wave numbers we consider in the present study. The viscosity is mainly related to the ion dynamics, and the kinematic viscosity terms may be calculated using the parallel and perpendicular viscosity coefficients, $\mu_{z}=\left[0.406\left(4 \pi \varepsilon_{0}\right)^{2} m_{\mathrm{i}}^{1 / 2}\left(\kappa T_{\mathrm{i}}\right)^{5 / 2}\right] /\left(z^{4} e^{4} \Lambda\right.$ ) (Mitchner \& Kruger 1973) and $\mu_{\perp}=\mu_{z} v_{\mathrm{ii}}^{2} / \Omega_{\mathrm{i}}^{2}$ (Spitzer 1962), respectively. Here, $v_{\mathrm{ii}}=4 \sqrt{\pi / m_{\mathrm{i}}}\left[e_{\mathrm{i}}^{2} /\left(4 \pi \varepsilon_{0}\right)\right]^{2} n_{\mathrm{i}} \Lambda / 3\left(\kappa T_{\mathrm{i}}\right)^{3 / 2}$ is ion-ion collision frequency, and $\Lambda$ denotes the Coulomb logarithm.

We consider the limiting case of when the plasma $\beta$ is higher than the electron-ion mass ratio, i.e. $\beta>m_{\mathrm{e}} / m_{\mathrm{i}}$, and the Alfvén wave speed is much lower than the electron thermal speed, so that the electron inertia is negligible. The approximation also implies that the electron-neutral collision frequency $v_{\mathrm{en}}$ is much higher than the wave frequency $\omega$.
In the equilibrium, from Eqs. (4) and (5) an analytical expression for the charge separation electric field is obtained in terms of the velocity of the neutral convection, $\boldsymbol{v}_{\mathrm{n}}$ :

$$
\begin{aligned}
e \boldsymbol{E}_{0} \approx & -\boldsymbol{v}_{\mathrm{n}} m_{\mathrm{i}} v_{\mathrm{in}}\left(\frac{\Omega_{\mathrm{e}} \Omega_{\mathrm{i}}}{v_{\mathrm{en}} v_{\mathrm{in}}} \frac{1}{1+v_{\mathrm{ei}} / v_{\mathrm{en}}}-\frac{\Omega_{\mathrm{i}}^{2}}{v_{\mathrm{in}}^{2}} \frac{1}{1+v_{\mathrm{en}} / v_{\mathrm{ei}}}\right) \\
& \times\left(1+\frac{\Omega_{\mathrm{e}} \Omega_{\mathrm{i}}}{v_{\mathrm{en}} v_{\mathrm{in}}} \frac{1}{1+v_{\mathrm{ei}} / v_{\mathrm{en}}}-\frac{\Omega_{\mathrm{i}}^{2}}{v_{\mathrm{in}}^{2}} \frac{1}{1+v_{\mathrm{en}} / v_{\mathrm{ei}}}\right)^{-1},
\end{aligned}
$$

using the inequalities (1) and (2) and the condition for the ambipolar diffusion, i.e. when the electrons and ions have the same velocity. In the case when the collisions with neutrals dominate the collisions among charged particles, expression (7) reduces to $e \boldsymbol{E}_{0} \approx-\boldsymbol{v}_{\mathrm{n}} m_{\mathrm{i}} v_{\mathrm{in}}$ (Sen \& White 1972). In the solar photosphere, the magnetic field is mainly vertical and, hence, we here consider a uniform magnetic field oriented in the $z$-direction, $\boldsymbol{B}_{0}=B_{0} \boldsymbol{e}_{z}$.

In principle, an additional equilibrium magnetic field is produced by the mentioned equilibrium electron drift induced by the neutral flow, given by

$\nabla \times \boldsymbol{B}_{0}^{\prime}=\mu_{0} \dot{j}_{0}$

This additional magnetic field causes magnetic shear, it is selfinduced, and its importance depends on the ratio $B_{0}^{\prime} / B_{0}$. Here, we consider length scales in the perpendicular direction to be much smaller than the characteristic magnetic shear length scale, so that this self-induced magnetic shear will be omitted. Furthermore, the equilibrium electron drift is supposed to be homogeneous, in the perpendicular direction, and denoted by $\boldsymbol{v}_{0} \equiv \boldsymbol{v}_{0 \perp}$. This also implies that the spatial inhomogeneity of the equilibrium electric field $\boldsymbol{E}_{0}$ is neglected and, correspondingly, the time variation of the magnetic field $\boldsymbol{B}_{0}$ as well. Formally this can be described by applying the operator $\nabla \times$ on the full Ampère law with the displacement current and using the Faraday law. This yields the limit used here $\left|\left[\left(\partial^{2} \boldsymbol{B} / \partial t^{2}\right)\right] /\left(c^{2} \nabla \boldsymbol{B}\right)\right| \ll 1$, i.e., $L^{2} / T^{2} \ll c^{2}$, where $L, T$ are the characteristic equilibrium inhomogeneity length and time. The corresponding expression in the perturbed state is $\omega^{2} / k^{2} \ll c^{2}$.

The perturbations of the magnetic and the electric fields can be expressed through their corresponding potentials. Considering only the bending of the magnetic field lines (since the stretching of the field lines demands more energy), these expressions take the following form: $\boldsymbol{B}_{1}=\boldsymbol{B}_{1 \perp}=\nabla_{\perp} A_{1 z} \times \boldsymbol{e}_{z}$, and $\boldsymbol{E}_{1}=-\nabla \phi_{1}-\partial \boldsymbol{A}_{1 z} / \partial t$. Here, $A_{1 z}$ denotes the $z$-component of the perturbed vector potential and $\phi_{1}$ the perturbed scalar potential of the electro-magnetic field.

In the low-frequency regime, i.e., $\omega \ll \Omega_{\text {i }}$, which is considered here, the ion perpendicular velocity perturbation is obtained from the ion momentum equation:

$$
\begin{aligned}
\boldsymbol{v}_{\mathrm{i} 1 \perp}= & \frac{\Omega_{\mathrm{i}}^{2}}{\Omega_{\mathrm{i}}^{2}+v_{\mathrm{in}}^{2}}\left[\frac{\boldsymbol{e}_{z} \times \nabla_{\perp} \phi}{B_{0}}+\frac{v_{\mathrm{Ti}}^{2}}{\Omega_{\mathrm{i}} n_{\mathrm{i}}} \boldsymbol{e}_{z} \times \nabla_{\perp} n_{\mathrm{i}}+\frac{\boldsymbol{e}_{z} \times \nabla_{\perp} \pi_{\mathrm{i}}}{e B_{0} n_{\mathrm{i}}}\right. \\
& \left.-\frac{1}{\Omega_{\mathrm{i}}}\left(\frac{\partial}{\partial t}+\boldsymbol{v} \cdot \nabla\right) \boldsymbol{e}_{z} \times \boldsymbol{v}_{\mathrm{i}}-\frac{v_{\mathrm{in}}}{\Omega_{\mathrm{i}} B_{0}} \nabla_{\perp} \phi-\frac{v_{\mathrm{in}} \rho_{\mathrm{i}}^{2}}{n_{\mathrm{i}}} \nabla_{\perp} n_{\mathrm{i}}\right]
\end{aligned}
$$

For the hot ions we have to include the effect of the finite Larmor radius. Hence, the diamagnetic contribution to the polarization drift and the stress tensor drift should be taken into account. The convective diamagnetic contribution to the polarization drift, $\boldsymbol{v}_{\mathrm{p}}$, in the term $\nabla\left(n v_{\mathrm{p}}\right)$ is cancelled by the part of the stress tensor contribution, $\nabla\left(n v_{\pi}\right)$, to the second order of small terms. The physical meaning of this cancellation is in the fact that the 
diamagnetic drift is not a particle drift and cannot transfer information by convection (Weiland 2000). The remainder of the term $\nabla\left(n \boldsymbol{v}_{\pi}\right)$, proportional to $\nabla \cdot \boldsymbol{v}$, for relatively long perpendicular wave-lengths (exceeding the ion gyro-radius by a lot) is usually negligible. However, for hot ions and shorter perpendicular scales, it approaches the polarization drift contribution and should be included in derivations. Taking this into account yields the terms

$n \rho_{\mathrm{i}}^{4} \nabla_{\perp}^{4} \frac{\partial}{\partial t}\left(\frac{e \phi_{1}}{T_{\mathrm{i}}}+\frac{n_{1}}{n_{0}}\right)$

in Eq. (6).

\section{Linear analysis}

We now discuss the results of the linear normal mode analysis we performed on the system described in the previous section. We first note that for the homogeneous plasma case, the perpendicular electron motion may be neglected completely, and because $\omega \ll k_{\|} C_{\mathrm{s}}$, the parallel ion motion can also be neglected (Weiland 2000).

Considering only small-amplitude perturbations, Eqs. (3)-(6) can be linearized. In the limit where the wave phase velocities are much smaller than the light speed, it is possible to neglect the displacement current in Ampére's law, which then reduces to:

$j_{1 z} \approx-\frac{1}{\mu_{0}} \triangle A_{1 z}$.

We also consider modes that have a very small variation along the magnetic field, i.e. $\nabla_{z} \ll \nabla_{\perp}$. Using the standard normal mode analysis, assuming that all perturbations can be decomposed in simple waves of the form $\sim \exp \left(-\mathrm{i} \omega t+\mathrm{i} k_{z} z+\mathrm{i} k_{\perp} r_{\perp}\right)$, the following linear system of equations is obtained for the perturbed quantities $v_{e 1 z}, n_{1}, A_{1 z}$, and $\phi_{1}$ :

$v_{e 1 z}=\frac{1}{k_{z}}\left(\omega-k_{\perp} v_{0}\right) \frac{n_{1}}{n_{0}}$,

$-\mathrm{i} \frac{e k_{z}}{m_{\mathrm{e}}} \phi_{1}+\mathrm{i} A_{1 z} \frac{e}{m_{\mathrm{e}}}\left(\omega-k_{\perp} v_{0}\right)+\mathrm{i} k_{z} v_{\mathrm{Te}}^{2} \frac{n_{1}}{n_{0}}+\left(v_{\mathrm{en}}+v_{\mathrm{ei}}\right) v_{e 1 z}=0,($

$A_{1 z} \frac{k_{z} k_{\perp}^{2}}{\mu_{0} e n_{0}}-\left\{k_{\perp}^{2} \rho_{\mathrm{i}}^{2}\left[\omega\left(1+k_{\perp}^{2} \rho_{\mathrm{i}}^{2}\right)+\mathrm{i} v_{\mathrm{in}}\right]+k_{\perp} v_{0}\right\} \frac{n_{1}}{n_{0}}$

$-k_{\perp}^{2} \rho_{\mathrm{i}}^{2}\left[\omega\left(1+k_{\perp}^{2} \rho_{\mathrm{i}}^{2}\right)+i v_{\mathrm{in}}\right] \frac{e \phi_{1}}{\kappa T_{\mathrm{i}}}=0$

$v_{e 1 z}=-\frac{k_{\perp}^{2}}{e n_{0} \mu_{0}} A_{1 z}$

From the set of Eqs. (11)-(14), the following dispersion equation can be derived in a straightforward manner:

$$
\begin{aligned}
& \left(\omega-k_{\perp} v_{0}\right)^{2}\left[\omega\left(1+k_{\perp}^{2} \rho_{\mathrm{i}}^{2}\right)+\mathrm{i} v_{\mathrm{in}}\right] \\
& -k_{z}^{2} v_{\mathrm{A}}^{2}\left\{\left[\omega\left(1+k_{\perp}^{2} \rho_{\mathrm{i}}^{2}\right)+\mathrm{i} v_{\mathrm{in}}\right] k_{\perp}^{2}\left(\rho_{\mathrm{s}}^{2}+\rho_{\mathrm{i}}^{2}\right)+\omega\right\} \\
& +\mathrm{i}\left(v_{\mathrm{en}}+v_{\mathrm{ei}}\right)\left[\omega\left(1+k_{\perp}^{2} \rho_{\mathrm{i}}^{2}\right)+\mathrm{i} v_{\mathrm{in}}\right]\left(\omega-k_{\perp} v_{0}\right) k_{\perp}^{2} \rho_{\mathrm{s}}^{2} \frac{v_{\mathrm{A}}^{2}}{v_{\mathrm{Te}}^{2}}=0,
\end{aligned}
$$

where

$\rho_{\mathrm{s}}^{2}=\frac{\kappa T_{\mathrm{e}}}{m_{\mathrm{i}} \Omega_{\mathrm{i}}^{2}}, \quad \rho_{\mathrm{i}}^{2}=\frac{\kappa T_{\mathrm{i}}}{m_{\mathrm{i}} \Omega_{\mathrm{i}}^{2}}, \quad$ and $\quad v_{\mathrm{A}}^{2}=\frac{B_{0}^{2}}{n_{0} m_{\mathrm{i}} \mu_{0}}$.
This dispersion relation describes the coupled drift-driven and dispersive Alfvén modes in a strongly collisional plasma.

By assuming $\omega=\omega_{\mathrm{r}}+\mathrm{i} \omega_{\mathrm{i}}$, with $\omega_{\mathrm{i}} \ll \omega_{\mathrm{r}}$, it is possible to separate the real and the imaginary parts in Eq. (15) yielding for the real part of the frequency (where small terms $\sim \omega_{\mathrm{i}}$ have been neglected)

$\omega_{\mathrm{r}}\left(\omega_{\mathrm{r}}-k_{\perp} v_{0}\right)^{2}\left(1+k_{\perp}^{2} \rho_{\mathrm{i}}^{2}\right)$

$-\omega_{\mathrm{r}} k_{\|}^{2} v_{\mathrm{A}}^{2}\left[1+\rho_{\mathrm{s}}^{2} k_{\perp}^{2}\left(1+\frac{T_{\mathrm{i}}}{T_{\mathrm{e}}}\right)\left(1+k_{\perp}^{2} \rho_{\mathrm{i}}^{2}\right)\right]$

$-v_{\text {in }}\left(v_{\mathrm{en}}+v_{\mathrm{ei}}\right)\left(\omega_{\mathrm{r}}-k_{\perp} v_{0}\right) k_{\perp}^{2} \rho_{\mathrm{s}}^{2} \frac{v_{\mathrm{A}}^{2}}{v_{\mathrm{Te}}^{2}}=0$,

and

$$
\begin{aligned}
\omega_{\mathrm{i}}= & -\left\{\left(v_{\mathrm{en}}+v_{\mathrm{ei}}\right)\left(\omega_{\mathrm{r}}-k_{\perp} v_{0}\right)\left[\omega_{\mathrm{r}}^{2}\left(1+k_{\perp}^{2} \rho_{\mathrm{i}}^{2}\right)^{2}+v_{\mathrm{in}}^{2}\right] k_{\perp}^{2} \rho_{\mathrm{s}}^{2} \frac{v_{\mathrm{A}}^{2}}{v_{\mathrm{Te}}^{2}}\right. \\
& \left.+v_{\mathrm{in}} \omega_{\mathrm{r}} k_{\|}^{2} v_{\mathrm{A}}^{2}\right\}\left\{( 1 + k _ { \perp } ^ { 2 } \rho _ { \mathrm { i } } ^ { 2 } ) \left[2 \omega_{\mathrm{r}}^{2}\left(\omega_{\mathrm{r}}-k_{\perp} v_{0}\right)\left(1+k_{\perp}^{2} \rho_{\mathrm{i}}^{2}\right)\right.\right. \\
& \left.\left.-v_{\mathrm{in}}\left(v_{\mathrm{en}}+v_{\mathrm{ei}}\right) k_{\perp} v_{0} k_{\perp}^{2} \rho_{\mathrm{s}}^{2} v_{\mathrm{A}}^{2} / v_{\mathrm{Te}}^{2}\right]\right\}^{-1}
\end{aligned}
$$

for the imaginary part.

\section{Discussion}

In general, the drift motion of particles is associated with a collective mode in the system (Ichimaru 1980), given by

$\omega=\boldsymbol{k} \cdot \boldsymbol{v}_{0}$,

where $\boldsymbol{v}_{0}$ denotes the drift velocity. For the collision-less case, i.e., in the case $v_{\mathrm{en}}=v_{\mathrm{ei}}=v_{\mathrm{in}}=0$, Eq. (15) simplifies to:

$\left(\omega-k_{\perp} v_{0}\right)^{2}-k_{z}^{2} v_{\mathrm{A}}^{2}\left(1+\rho_{\mathrm{s}}^{2} k_{\perp}^{2}\right)=0$.

Hence, in this case the classical kinetic Alfvén mode, $\omega^{2}=$ $k_{z}^{2} v_{\mathrm{A}}^{2}\left(1+\rho_{\mathrm{s}}^{2} k_{\perp}^{2}\right)$, is modified due to this collective drift mode.

In the fluid theory and in a collision-less plasma, the electron drift gives thus rise to a drift-driven (collective) mode and a modification of the electromagnetic modes present in the system, here, in particular, the kinetic Alfvén wave. Note that there is no instability in a collision-less plasma even if there is an electron drift present. In contrast, in the kinetic plasma theory, if there is an electron drift present in the system, i.e. when the electron distribution function is shifted relative to the ion distribution function, this may cause a destabilization of the system when the drift velocity exceeds some threshold value. In this case, the electron drift is the source supplying the necessary energy for the instability. In the fluid description of the plasma dynamics, however, the presence of collisions is a necessary condition for an instability.

From Eq. (17) it is clear that electron collisions lead to damping of the kinetic Alfvén mode. As a matter of fact, ignoring the ion collisions and the electron drift, one easily obtains the decrement of the KAW, in this case: $\omega_{\mathrm{i}} \sim-\left(v_{\mathrm{en}}+v_{\mathrm{ei}}\right) e k_{\perp}^{2} \rho_{\mathrm{s}}^{2} v_{\mathrm{A}}^{2} / v_{\mathrm{Te}}^{2}$. Without the drift in the system, the ion collisions also result in the damping of the kinetic Alfvén mode. For $k_{\perp}^{2} \lambda_{\mathrm{e}}^{2} \ll 1$, we obtain the decrement $\omega_{\mathrm{i}} \sim-v_{\text {in }} / 2$.

Equation (15) is rather complicated, although it is only of the third order, and, in general, calculating and discussing the exact solutions of this equation is not easy. However, to some 
extent the character of the solutions may be understood even without directly solving the dispersion equation, viz. by using the generalized Hurwitz method for polynomials with complex coefficients (Giaretta 1979). As a matter of fact, the dispersion relation (15) is a polynomial equation. For a general polynomial in $x$, of degree $m$, and with complex coefficients,

$a_{0} x^{m}+\left(a_{1}+\mathrm{i} b_{1}\right) x^{m-1}+\ldots+\left(a_{m}+\mathrm{i} b_{m}\right)=0$,

the number of roots with positive real parts equals the number of sign changes in the sequence $c_{0}, c_{1}, \ldots, c_{m}$ (Giaretta 1979), where $c_{0}=a_{0}, c_{1}=a_{1}$, and

$c_{\mathrm{r}}=(-1)^{r(r-1) / 2}\left|\begin{array}{cccccc}a_{1} & a_{0} & 0 & 0 & 0 & \cdots \\ -b_{2} & -b_{1} & a_{1} & a_{0} & 0 & \cdots \\ a_{3} & a_{2} & b_{2} & b_{1} & a_{1} & \cdots \\ \vdots & \vdots & \vdots & \vdots & \vdots & \\ a_{2 r-1} & a_{2 r-2} & b_{2 r-2} & b_{2 r-3} & a_{2 r-3} & \cdots\end{array}\right|$,

to $r=m$. Thus, rewriting Eq. (15) in the required form, a sufficient condition for an instability to exist is at least one sign change in the sequence:

$c_{0}=\frac{e^{2} k_{\perp}^{2} \rho_{\mathrm{i}}^{2}}{m_{\mathrm{e}}}\left(1+k_{\perp}^{2} \rho_{\mathrm{i}}^{2}\right), \quad c_{1}=-2 \boldsymbol{k}_{\perp} \cdot \boldsymbol{v}_{0} c_{0}, \ldots$

From the first two terms in this sequence, the generalized Hurwitz method leads to the conclusion that, if $\boldsymbol{k}_{\perp} \cdot \boldsymbol{v}_{0}$ is positive, there is an instability in the system.

In the photosphere, most of the electrons arise from metal ionization because only a very small fraction of the photospheric hydrogen, $H$, is ionized. As a matter of fact, the degree of $H$ ionization in the lower solar atmosphere starts from $3 \times 10^{-4}$, at an altitude of $1 \mathrm{~km}$, then decreases to reach a minimum, $2 \times 10^{-7}$, at $500 \mathrm{~km}$ (the top of the photosphere), and then rises again to 1 at an altitude of $2000 \mathrm{~km}$ (the top of the chromosphere) (Priest 1987). The mean molecular weight of metal ions in the photosphere can be assumed to be 35 of the hydrogen molecular weight (Sen \& White 1972).

We solved Eq. (15) numerically for physical parameter values that are typical of the solar photosphere at an altitude of $350 \mathrm{~km}$ Vernazza et al. (1981), i.e. $n_{\mathrm{n}}=9.9 \times 10^{21} \mathrm{~m}^{-3}$, $n_{0}=1.1 \times 10^{18} \mathrm{~m}^{-3}, T_{\mathrm{i}}=T_{\mathrm{e}}=4400 \mathrm{~K}$, and $B=0.025 \mathrm{~T}$. In Fig. 1, the resulting imaginary part of the frequency (in $\mathrm{Hz}$ ) of the electron-drift driven mode is shown versus the drift velocity (in $\mathrm{m} / \mathrm{s}$ ) for $k_{\perp}=0.5 \mathrm{~m}^{-1}$. Clearly, a cross-field electron drift in a collisional plasma can give rise to an instability in the system when a certain critical or threshold drift velocity is exceeded. For the case discussed here, this threshold drift velocity amounts to $\sim 400 \mathrm{~m} / \mathrm{s}$, which seems to be consistent with the observed range of the plasma flow velocities in the solar photosphere. As a matter of fact, a drift velocity of this magnitude can be generated by an electric field of $\sim 10 \mathrm{~V} / \mathrm{m}$. According to relation (7), a convective neutral velocity field of magnitude $\sim 1.1 \mathrm{~km} \mathrm{~s}^{-1}$ is enough to produce such a field and thus cause an instability in the system. Velocities of this order of magnitude are observed in the solar photosphere. The real part of frequency of the electron drift driven mode is on the order $10^{2} \mathrm{~Hz}$.

The electron-neutral, ion-neutral, and electron-ion collision frequencies are calculated from the expressions:

$$
\begin{aligned}
& v_{\mathrm{en}}=\sigma n_{\mathrm{n}} \sqrt{8 \kappa \frac{T_{\mathrm{e}}}{\pi m_{\mathrm{e}}}}, \\
& v_{\mathrm{in}}=\sigma n_{\mathrm{n}} \frac{m_{\mathrm{n}}}{m_{\mathrm{n}}+m_{\mathrm{i}}} \sqrt{\frac{8 \kappa T_{\mathrm{e}}\left(m_{\mathrm{i}}+m_{\mathrm{n}}\right)}{\pi m_{\mathrm{i}} m_{\mathrm{n}}}},
\end{aligned}
$$

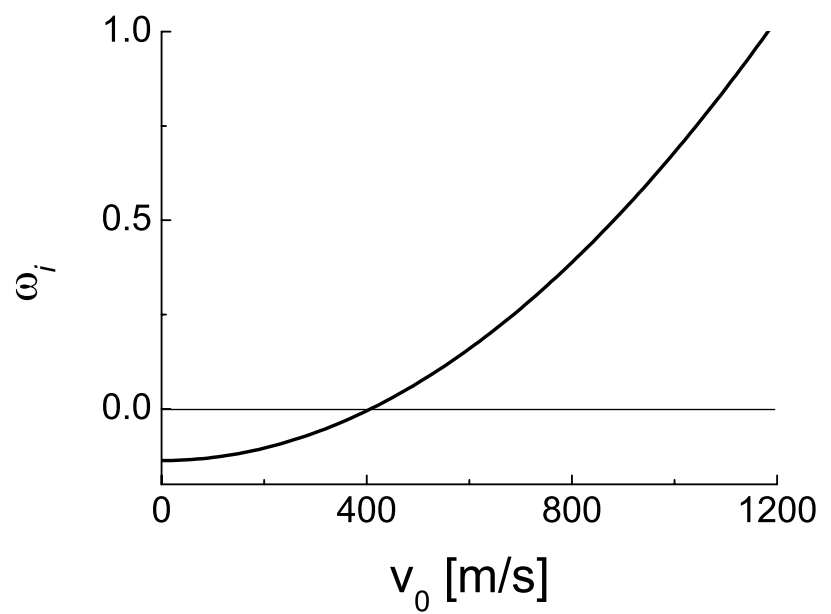

Fig. 1. The imaginary part of the frequency (in $\mathrm{Hz}$ ) of the electron-drift driven mode as a solution of Eq. (15) versus the drift velocity (in $\mathrm{m} / \mathrm{s}$ ) for $k_{\perp} / k_{\|}=500$ for the solar photosphere plasma parameters given in the text.

and

$v_{\mathrm{ei}}=4 \sqrt{2 \pi / m_{\mathrm{e}}}\left[e e_{\mathrm{i}} /\left(4 \pi \varepsilon_{0}\right)\right]^{2} n_{\mathrm{e}} L / 3\left(\kappa T_{\mathrm{e}}\right)^{3 / 2}$,

where $\sigma=0.88 \times 10^{-20} \mathrm{~m}^{2}$, denotes the electron-neutral collision cross section (Chen 1984).

In a strongly collisional system like the relatively dense solar photosphere, the kinetic Alfvén mode is totally damped and cannot exist without cross-field electron drift. A finite electron drift decreases the influence of the collisions and thus results in a weaker damping of the mode, enabling its appearance in the system. However, the kinetic Alfvén mode is still damped and cannot become unstable due to this electron drift. In Fig. 2 we display the $\left|\omega_{i}\right| / \omega_{\text {r }}$ ratio for one branch of the kinetic Alfvén wave as a solution of Eq. (15) versus the drift velocity (in $\mathrm{m} / \mathrm{s}$ ) for the photospheric plasma parameters mentioned above. Clearly, the KAW is damped for all values of the drift velocity, but for higher drift velocities, the $\left|\omega_{\mathrm{i}}\right| / \omega_{\mathrm{r}}\left(=-\omega_{\mathrm{i}} / \omega_{\mathrm{r}}\right)$ ratio is much lower than for lower electron drift velocities, mainly due to the fact that $\omega_{\mathrm{r}}$ becomes finite in this case.

The instability of the system thus originates only from the electron-drift driven mode, i.e. the $k_{\perp} v_{0}$ mode, which is destabilized when the cross-field drift $v_{0}$ exceeds some critical threshold value. The instability is thus in fact generated by the difference in the electron and ion perpendicular drifts, while the electron motion along the magnetic field lines has a stabilizing influence by short-circuiting the charge separation (Weiland 2000).

Similarly, in an electrostatic study of the ionization effect on the electron-drift driven (i.e. the Farley-Buneman type) mode, it has been shown recently that even a small parallel wave number has considerable influence and can lead to a substantial increase in the instability threshold, and even to the complete stabilization of the plasma (Petrović et al. 2005). However, for sufficiently small parallel wave numbers, the ionization is the dominant effect in the system causing an instability.

In Fig. 3 the dependence of the real and imaginary parts (in $\mathrm{Hz}$, imaginary part $\times 10$ ) of the frequency of the electron-drift driven mode on $k_{\perp} \rho_{\mathrm{s}}$ is shown, again as a solution of Eq. (15) for the drift velocity $v_{0}=600 \mathrm{~m} / \mathrm{s}$ and $k_{\|}=0.01 \mathrm{~m}^{-1}$. The real part of the frequency increases with increasing $k_{\perp} \rho_{\mathrm{s}}$. For low values of $k_{\perp} \rho_{\mathrm{s}}$, the system is unstable but the growth rate of the instability is relatively low. For increasing $k_{\perp} \rho_{\mathrm{s}}$, the growth rate first increases, reaches a maximal value around $k_{\perp} \rho_{\mathrm{s}}=0.002$, and then 


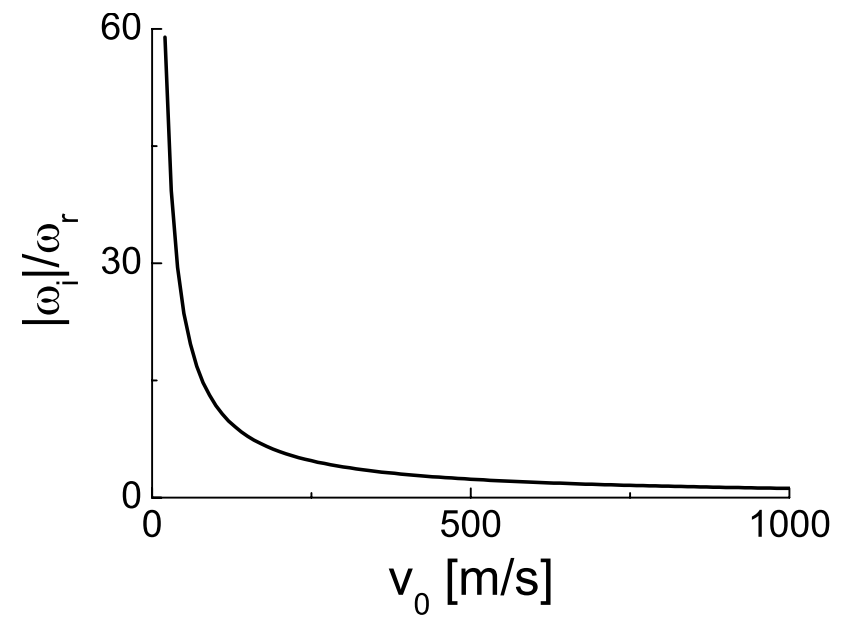

Fig. 2. The $\left|\omega_{\mathrm{i}}\right| / \omega_{\mathrm{r}}$ ratio for one branch of the kinetic Alfvén mode as a solution of Eq. (15) versus the drift velocity (in $\mathrm{m} / \mathrm{s}$ ) for the photospheric plasma.

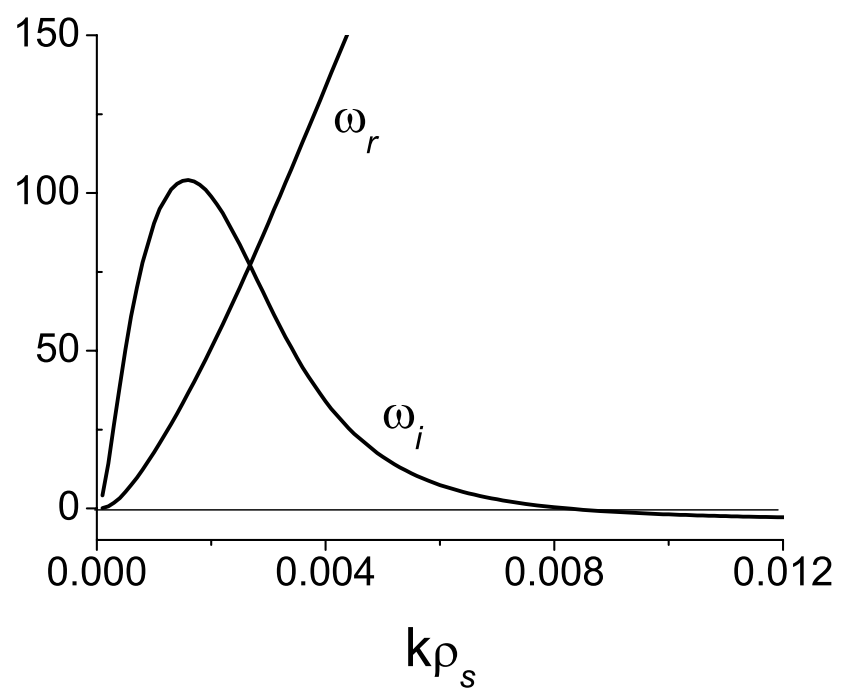

Fig. 3. The real $\omega_{\mathrm{r}}$ and imaginary (multiplied by 10) $\omega_{\mathrm{i}}$ part of the frequency (in $\mathrm{Hz}$ ) of the electron-drift driven mode in terms of $k_{\perp} \rho_{\mathrm{s}}$ for the drift velocity $v_{0}=600 \mathrm{~m} / \mathrm{s}$ and $k_{\|}=0.01 \mathrm{~m}^{-1}$.

decreases again to become negative at about $k_{\perp} \rho_{\mathrm{s}}=0.0085$. This behaviour is similar for other values of the drift velocity and the parallel wave number. For the illustration in Fig. 3, again parameter values have been chosen that are appropriate for the solar photospheric plasma for this quantitative illustration. However, we stress that the model and the equations derived in this paper are valid for any partially ionized plasma comprising a substantial amount of neutral atoms.

For instance, considering a pure hydrogen plasma system (without metal ions) with the same parameters, the instability threshold decreases and the threshold velocity is only $\sim 250 \mathrm{~m} / \mathrm{s}$ in this case. The corresponding, appropriate neutral convective velocity is only $\sim 0.7 \mathrm{~km} \mathrm{~s}^{-1}$, as is shown in Fig. 4 . In practice, this means that when the ions are less magnetized, their drift is smaller and the instability is thus driven more easily. The more unstable behaviour of the pure hydrogen plasma is not only clear from the lower threshold of the instability (in terms of the electron drift velocity) but also from the increased growth rates as compared to the case of a hydrogen plasma with metal ions, discussed above in the context of the solar photosphere and displayed again in Fig. 4 for comparison.

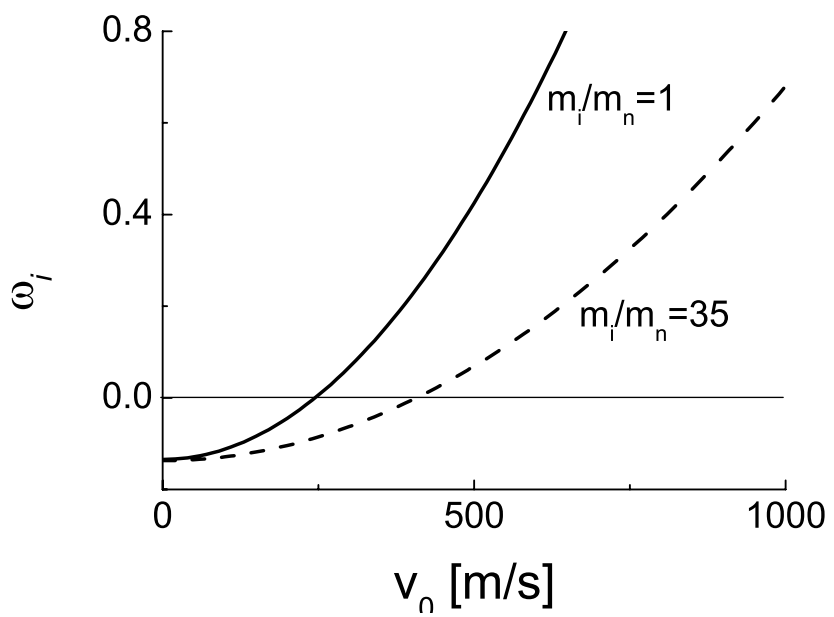

Fig. 4. The imaginary part of the frequency (in $\mathrm{Hz}$ ) for the electron-drift driven mode as a solution of Eq. (15) versus the drift velocity (in $\mathrm{m} / \mathrm{s}$ ) for the pure hydrogen plasma (full line) and for the hydrogen plasma with metal ions (dashed lined).

\section{Nonlinear analysis}

The perturbations in the solar photosphere are not always small. As a matter of fact, those in the solar atmosphere can be extremely violent, e.g. during a solar flare or a coronal mass ejection. For such events, the linear analysis presented and discussed in the previous two sections is not justified any more. We thus also performed a nonlinear analysis, assuming strong perturbations and keeping in mind that one of the modes in the system is unstable, hereby taking into account the electron perpendicular motion in the perturbed state. Since we are interested in nonlinear self-organization at scales far exceeding the mean free path of particles, from now on we omit the collisional terms.

The perpendicular velocity is obtained from the electron momentum equation taking into account that $\partial / \partial t \ll \Omega_{\mathrm{i}}$ and $\nabla_{z} \ll \nabla_{\perp}$, and neglecting the small terms of the third and higher orders:

$\boldsymbol{v}_{e 1 \perp}=\frac{1}{B_{0}} \boldsymbol{e}_{z} \times \nabla_{\perp}\left(\phi_{1}-\frac{B_{0} v_{\mathrm{Te}}^{2}}{\Omega_{\mathrm{e}} n_{0}} n\right)+\frac{1}{B_{0}} v_{e 1 z} \boldsymbol{B}_{1 \perp}$.

Looking for possible local nonlinear solutions traveling with a phase velocity $\boldsymbol{u}_{\mathrm{ph}}=u_{y} \boldsymbol{e}_{y}+u_{z} \boldsymbol{e}_{z}$ we can write

$$
\begin{aligned}
& \frac{\partial}{\partial t}=-u_{y} \frac{\partial}{\partial y}=-\frac{1}{B_{0}}\left[\boldsymbol{e}_{z} \times \nabla_{\perp}\left(B_{0} u_{y} x\right)\right] \nabla_{\perp}, \\
& \frac{\partial}{\partial z}=\frac{u_{y}}{u_{z}} \frac{\partial}{\partial y}=\frac{1}{B_{0}}\left[\boldsymbol{e}_{z} \times \nabla_{\perp}\left(B_{0} \frac{u_{y}}{u_{z}} x\right)\right] \nabla_{\perp}
\end{aligned}
$$

The nonlinear propagation is then described by the following system of partial differential equations:

$$
\begin{aligned}
& {\left[\boldsymbol{e}_{z} \times \nabla_{\perp}\left(\phi_{1}+\left(v_{0}-u_{y}\right) B_{0} x\right) \cdot \nabla_{\perp}\right] n_{1}} \\
& \quad+\frac{1}{\mu_{0} e}\left[\boldsymbol{e}_{z} \times \nabla_{\perp}\left(\frac{u_{y}}{u_{z}} B_{0} x-A_{1 z}\right) \cdot \nabla_{\perp}\right] \nabla_{\perp}^{2} A_{1 z}=0 \\
& \frac{1}{B_{0}}\left[\boldsymbol{e}_{z} \times \nabla_{\perp}\left(\phi_{1}-\frac{\kappa T_{\mathrm{e}}}{e n_{0}} n_{1}+\left(v_{0}-u_{y}\right) B_{0} x\right) \nabla_{\perp}\right] \\
& \quad \times\left(A_{1 z}-\frac{u_{y}}{u_{z}} B_{0} x\right)=0
\end{aligned}
$$


and

$$
\begin{aligned}
& \frac{1}{B_{0}}\left[\boldsymbol{e}_{z} \times \nabla_{\perp}\left(\phi_{1}-u_{y} B_{0} x\right) \cdot \nabla_{\perp}\right]\left(n_{0} \rho_{\mathrm{i}}^{2} \nabla_{\perp}^{2} \frac{e \phi_{1}}{T_{\mathrm{i}}}+\rho_{\mathrm{i}}^{2} \nabla_{\perp}^{2} n_{1}\right) \\
& +\frac{1}{\mu_{0} e B_{0}}\left[\boldsymbol{e}_{z} \times \nabla_{\perp}\left(\frac{u_{y}}{u_{z}} B_{0} x-A_{1 z}\right) \cdot \nabla_{\perp}\right] \nabla_{\perp}^{2} A_{1 z} \\
& -\frac{1}{B_{0}}\left[\boldsymbol{e}_{z} \times \nabla_{\perp}\left(v_{0} B_{0} x\right) \cdot \nabla_{\perp}\right] n_{1}=0,
\end{aligned}
$$

which are the electron continuity equation, the parallel electron momentum equation, and the quasi-neutrality equation, respectively. In Eq. (25), the terms $\sim \rho_{\mathrm{s}}^{4} \nabla_{\perp}^{4}$ have been neglected as these are small compared to the scale of the nonlinear coherent structures we are looking for.

Equation (24) has the form

$\left[\boldsymbol{e}_{z} \times \nabla_{\perp} A(x, y)\right] \nabla_{\perp} B(x, y)=0$,

and thus has a general solution $B=F(A)$, where $F$ is an arbitrary function of the given argument. After integration we obtain:

$A_{1 z}-\frac{u_{y}}{u_{z}} B_{0} x=F\left(\phi_{1}-\frac{\kappa T_{\mathrm{e}}}{e n_{0}} n_{1}+\left(v_{0}-u_{y}\right) B_{0} x\right)$.

Taking the function $F$ as linear, i.e.

$F(\xi)=F_{1} \cdot \xi+F_{2}$,

and using the condition of vanishing perturbations at infinity, we obtain

$A_{1 z}=F_{1} \cdot\left(\phi_{1}-\frac{\kappa T_{\mathrm{e}}}{e n_{0}} n_{1}\right)$

and

$\frac{u_{y}}{u_{z}}=-F_{1} \cdot\left(v_{0}-u_{y}\right)$.

By combining Eqs. (23) and (25), with a similar procedure we obtain:

$n_{0} \rho_{\mathrm{i}}^{2} \nabla_{\perp}^{2} \frac{e \phi_{1}}{T_{\mathrm{i}}}+\rho_{\mathrm{i}}^{2} \nabla_{\perp}^{2} n_{1}-n_{1}=D\left(\phi_{1}-B_{0} u_{y} x\right)$

Using Eq. (27), from Eq. (23), the following equation is obtained:

$n_{1}-\frac{F_{1}^{2}}{e \mu_{0}} \nabla_{\perp}^{2}\left(\phi_{1}-\frac{\kappa T_{\mathrm{e}}}{e n_{0}} n_{1}\right)=G\left(\phi_{1}+\left(v_{0}-u_{y}\right) B_{0} x-\frac{\kappa T_{\mathrm{e}}}{e n_{0}} n_{1}\right)$.

Here, $D$ and $G$ are two additional arbitrary functions of the given argument, which we take to be linear as well, i.e.; we choose $D(\xi)=D_{1} \cdot \xi$, and $G(\xi)=G_{1} \cdot \xi$.
Using Eq. (29) in Eq. (30), the following differential equation for the electrostatic potential is obtained:

$$
\begin{aligned}
& \nabla_{\perp}^{4} \phi_{1}+\nabla_{\perp}^{2} \phi_{1}\left[\frac{G_{1}\left(\rho_{\mathrm{s}}^{2}+\rho_{\mathrm{i}}^{2}\right)}{F_{1}^{2} v_{\mathrm{A}}^{2} \rho_{\mathrm{s}}^{2}}-m_{\mathrm{i}} \Omega_{\mathrm{i}}^{2} D_{1}-\frac{e n_{0}}{\rho_{\mathrm{s}}^{2}}+\frac{e n_{0}}{F_{1}^{2} v_{\mathrm{A}}^{2} \rho_{\mathrm{s}}^{2}}\right] \\
& -\phi_{1} \frac{D_{1} m_{\mathrm{i}} \Omega_{\mathrm{i}}^{2}}{e n_{0} F_{1}^{2} v_{\mathrm{A}}^{2}\left(\rho_{\mathrm{s}}^{2}+\rho_{\mathrm{i}}^{2}\right)}\left(1+G_{1} \frac{\kappa T_{\mathrm{e}}}{e n_{0}}\right)-\frac{G_{1} m_{\mathrm{i}} \Omega_{\mathrm{i}}^{2} v_{0} B_{0} x}{e n_{0} F_{1}^{2} v_{\mathrm{A}}^{2}\left(\rho_{\mathrm{s}}^{2}+\rho_{\mathrm{i}}^{2}\right)} \\
& +\frac{u_{y} B_{0} x}{e n_{0} F_{1}^{2} v_{\mathrm{A}}^{2}\left(\rho_{\mathrm{s}}^{2}+\rho_{\mathrm{i}}^{2}\right)}\left[\left(G_{1}+D_{1}\right) m_{\mathrm{i}} \Omega_{\mathrm{i}}^{2}+G_{1} D_{1} m_{\mathrm{i}} \Omega_{\mathrm{i}}^{2} \frac{\kappa T_{\mathrm{e}}}{e n_{0}}\right]=0 .
\end{aligned}
$$

Equation (31) can be rewritten in the form

$$
\left(\nabla_{\perp}^{2}+M_{1}^{2}\right)\left(\nabla_{\perp}^{2}+M_{2}^{2}\right)\left(\phi_{1}+\frac{c x}{M_{1}^{2} M_{2}^{2}}\right)=0
$$

where the constants $M_{1}$ and $M_{2}$ should be found from the following set of equations:

$M_{1}^{2}+M_{2}^{2}=\left[\frac{G_{1}\left(\rho_{\mathrm{s}}^{2}+\rho_{\mathrm{i}}^{2}\right)}{F_{1}^{2} v_{\mathrm{A}}^{2} \rho_{\mathrm{s}}^{2}}-m_{\mathrm{i}} \Omega_{\mathrm{i}}^{2} D_{1}-\frac{e n_{0}}{\rho_{\mathrm{s}}^{2}}+\frac{e n_{0}}{F_{1}^{2} v_{\mathrm{A}}^{2} \rho_{\mathrm{s}}^{2}}\right]$,

$M_{1}^{2} M_{2}^{2}=\frac{D_{1} m_{\mathrm{i}} \Omega_{\mathrm{i}}^{2}}{e n_{0} F_{1}^{2} v_{\mathrm{A}}^{2}\left(\rho_{\mathrm{s}}^{2}+\rho_{\mathrm{i}}^{2}\right)}\left(1+G_{1} \frac{\kappa T_{\mathrm{e}}}{e n_{0}}\right)$,

and where

$$
\begin{aligned}
c= & -\frac{G_{1} m_{\mathrm{i}} \Omega_{\mathrm{i}}^{2} v_{0} B_{0}}{e n_{0} F_{1}^{2} v_{\mathrm{A}}^{2}\left(\rho_{\mathrm{s}}^{2}+\rho_{\mathrm{i}}^{2}\right)} \\
& +\frac{u_{y} B_{0} m_{\mathrm{i}} \Omega_{\mathrm{i}}^{2}}{e n_{0} F_{1}^{2} v_{\mathrm{A}}^{2}\left(\rho_{\mathrm{s}}^{2}+\rho_{\mathrm{i}}^{2}\right)}\left[G_{1}+D_{1}+G_{1} D_{1} \frac{\kappa T_{\mathrm{e}}}{e n_{0}}\right] .
\end{aligned}
$$

Equation (32) can be solved in cylindrical coordinates. Dividing the space by an arbitrary circle of radius $r_{0}$, the solution can be determined inside this circle, i.e., for $r<r_{0}$ and outside $(r>$ $r_{0}$ ) of the circle. This will yield inside and outside values for the integration constants and thus also for the constants $c$ and $M_{1}$ and $M_{2}$, defined above. This standard method (Larichev \& Reznik 1976; Vranjes 1999) yields, respectively,

$\phi_{1}^{\text {in }}(r, \theta)=\left[\alpha_{2} J_{1}\left(\kappa_{2} r\right)-\frac{\alpha_{1}}{\kappa_{1}^{2}-\kappa_{2}^{2}} J_{1}\left(\kappa_{1} r\right)-\frac{c^{\text {in }} r}{\kappa_{1}^{2} \kappa_{2}^{2}}\right] \cos \theta$,

and

$\phi_{1}^{\text {out }}(r, \theta)=\left[\beta_{2} K_{1}\left(\lambda_{2} r\right)-\frac{\beta_{1}}{\lambda_{2}^{2}-\lambda_{1}^{2}} K_{1}\left(\lambda_{1} r\right)\right] \cos \theta$,

where $J_{1}$ and $K_{1}$ are the Bessel function and the modified Bessel function of the second kind, respectively, $\kappa_{1(2)}^{2}=\left(M_{1(2)}^{\text {in }}\right)^{2}$, and $\lambda_{1(2)}^{2}=-\left(M_{1(2)}^{\text {out }}\right)^{2}$. The constant $c^{\text {out }}=0$ because $\phi$ is zero at infinity. This gives us a connection between the integration constants $D_{1}^{\text {out }}$ and $G_{1}^{\text {out }}$

$G_{1}^{\text {out }}\left(v_{0}-u_{y}\right)=D_{1}^{\text {out }}\left(1+G_{1}^{\text {out }} \frac{\kappa T_{\mathrm{e}}}{e n_{0}}\right) u_{y}$,

while in the inner area there are no extra conditions. 
Knowing the scalar potential $\phi_{1}$ from Eq. (29), with a similar procedure, the number density $n_{1}$ can be determined. This yields

$n_{1}^{\text {in }}(r, \theta)=\left[\gamma_{1} J_{1}(\chi r)-\frac{a J_{1}\left(\kappa_{2} r\right)}{\kappa_{2}^{2}-\chi^{2}}-\frac{b J_{1}\left(\kappa_{1} r\right)}{\kappa_{1}^{2}-\chi^{2}}+\frac{\mathrm{d} r}{\chi^{2}}\right] \cos \theta$,

$n_{1}^{\text {out }}(r, \theta)=\left[\delta_{1} K_{1}\left(r / \rho_{\mathrm{i}}\right)+\frac{p K_{1}\left(\lambda_{2} r\right)}{\lambda_{2}^{2}-1 / \rho_{\mathrm{i}}^{2}}+\frac{q K_{1}\left(\lambda_{1} r\right)}{\lambda_{1}^{2}-1 / \rho_{\mathrm{i}}^{2}}\right] \cos \theta$,

where

$a=\alpha_{2}\left(\frac{e n_{0} \kappa_{2}^{2}}{T_{\mathrm{i}}}+\frac{D_{1}^{\mathrm{in}}}{\rho_{\mathrm{i}}^{2}}\right), \quad d=-D_{1}^{\mathrm{in}}\left(\frac{c^{\mathrm{in}}}{\kappa_{1}^{2} \kappa_{2}^{2}}+\frac{B_{0} u_{y}}{\rho_{\mathrm{i}}^{2}}\right)$,

$b=-\alpha_{1}\left[\frac{e n_{0} \kappa_{1}^{2}}{T_{\mathrm{i}}\left(\kappa_{1}^{2}-\kappa_{2}^{2}\right)}+\frac{D_{1}^{\mathrm{in}}}{\rho_{\mathrm{i}}^{2}\left(\kappa_{1}^{2}-\kappa_{2}^{2}\right)}\right]$,

$\chi^{2}=-\frac{1}{\rho_{\mathrm{i}}^{2}}, \quad p=-\lambda_{2}^{2} \beta_{2} \frac{e n_{0}}{T_{\mathrm{i}}}, \quad q=\frac{\lambda_{1}^{2} \beta_{1}}{\lambda_{2}^{2}-\lambda_{1}^{2}} \frac{e n_{0}}{T_{\mathrm{i}}}$.

Considering the condition of the vanishing perturbations at infinity, $D_{1}^{\text {out }}$ must be taken as equal to zero. Combined with the relations (28) and (35), this leads to $G_{1}^{\text {out }}=0$, because it cannot be that $u_{y}=v_{0}$, i.e.; the nonlinear solution cannot travel with the perpendicular phase velocity, $u_{y}$, equal to the electron drift velocity $v_{0}$. This would lead to a contradiction because of Eq. (28). Having found solutions for the scalar potential and the number density, from Eq. (27) it is easy to calculate the vector potential, $A_{1 z}$, as well.

In order to have a physically meaningful solution, at the boundary $r=r_{0}$, the continuity conditions must be satisfied. These lead to the system of equations from which the remaining unknown integration constants, $G_{1}^{\text {in }}, D_{1}^{\text {in }}, \alpha_{1}, \alpha_{2}, \beta_{1}, \beta_{2}, \gamma_{1}$, and $\delta_{1}$, and physical parameters, $r_{0}, u_{y}$, and $u_{z}$, can be determined.

The continuity of the potential $\phi_{1}$ at $r=r_{0}$ gives

$$
\begin{gathered}
\alpha_{2} J_{1}\left(\kappa_{2} r_{0}\right)-\frac{\alpha_{1}}{\kappa_{1}^{2}-\kappa_{2}^{2}} J_{1}\left(\kappa_{1} r_{0}\right)-\frac{c^{\text {in }} r_{0}}{\kappa_{1}^{2} \kappa_{2}^{2}}= \\
\beta_{2} K_{1}\left(\lambda_{2} r_{0}\right)-\frac{\beta_{1}}{\lambda_{2}^{2}-\lambda_{1}^{2}} K_{1}\left(\lambda_{1} r_{0}\right) .
\end{gathered}
$$

The continuity of the derivative of the potential $\phi_{1}$ with respect to $r$, at $r=r_{0}$ gives

$$
\begin{gathered}
\kappa_{2} \alpha_{2}\left[J_{0}\left(\kappa_{2} r_{0}\right)-\frac{J_{1}\left(\kappa_{2} r_{0}\right)}{\kappa_{2} r_{0}}\right]-\frac{\kappa_{1} \alpha_{1}}{\kappa_{1}^{2}-\kappa_{2}^{2}}\left[J_{0}\left(\kappa_{1} r_{0}\right)-\frac{J_{1}\left(\kappa_{1} r_{0}\right)}{\kappa_{1} r_{0}}\right] \\
-\frac{c^{\text {in }}}{\kappa_{1}^{2} \kappa_{2}^{2}}=-\lambda_{2} \beta_{2}\left[K_{0}\left(\lambda_{2} r_{0}\right)+\frac{K_{1}\left(\lambda_{2} r_{0}\right)}{\lambda_{2} r_{0}}\right] \\
+\frac{\lambda_{1} \beta_{1}}{\lambda_{2}^{2}-\lambda_{1}^{2}}\left[K_{0}\left(\lambda_{1} r_{0}\right)+\frac{K_{1}\left(\lambda_{1} r_{0}\right)}{\lambda_{1} r_{0}}\right] .
\end{gathered}
$$

The continuity of the Laplacian $\nabla^{2} \phi_{1}$ at $r=r_{0}$ gives

$$
\begin{aligned}
& -\kappa_{2}^{2} \alpha_{2} J_{1}\left(\kappa_{2} r_{0}\right)+\frac{\kappa_{1}^{2} \alpha_{1}}{\kappa_{1}^{2}-\kappa_{2}^{2}} J_{1}\left(\kappa_{1} r_{0}\right)= \\
& \lambda_{2}^{2} \beta_{2} K_{1}\left(\lambda_{2} r_{0}\right)-\frac{\lambda_{1}^{2} \beta_{1}}{\lambda_{2}^{2}-\lambda_{1}^{2}} K_{1}\left(\lambda_{1} r_{0}\right) .
\end{aligned}
$$

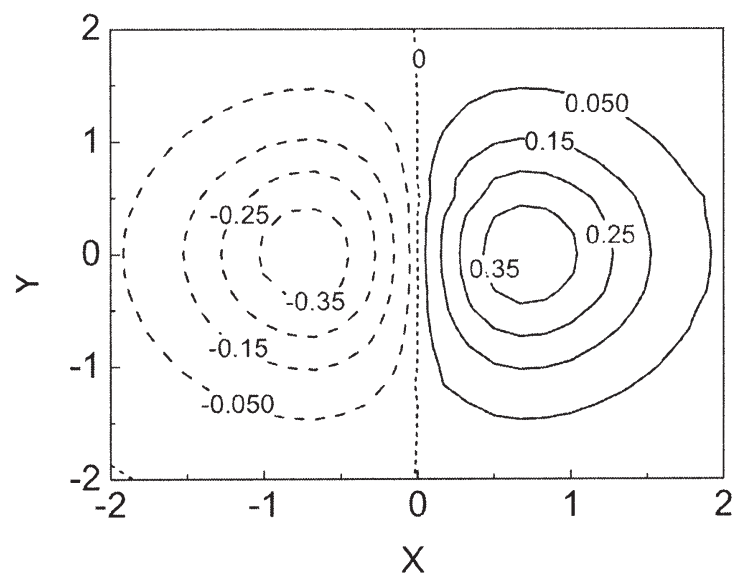

Fig. 5. The contour plot of the double vortex (in arbitrary units) as a sketch of the solution of Eq. (32). The negative vortex is represented by the dashed line and the positive vortex by the solid line.

The continuity of $n_{1}$ at $r=r_{0}$ gives

$$
\begin{gathered}
\gamma_{1} J_{1}\left(\chi r_{0}\right)-\frac{a J_{1}\left(\kappa_{2} r_{0}\right)}{\kappa_{2}^{2}-\chi^{2}}-\frac{b J_{1}\left(\kappa_{1} r_{0}\right)}{\kappa_{1}^{2}-\chi^{2}}+\frac{d r_{0}}{\chi^{2}}= \\
\delta_{1} K_{1}\left(r_{0} / \rho_{\mathrm{i}}\right)+\frac{p K_{1}\left(\lambda_{2} r_{0}\right)}{\lambda_{2}^{2}-1 / \rho_{\mathrm{i}}^{2}}+\frac{q K_{1}\left(\lambda_{1} r_{0}\right)}{\lambda_{1}^{2}-1 / \rho_{\mathrm{i}}^{2}} .
\end{gathered}
$$

The continuity of Laplacian $\nabla^{2} n_{1}$ at $r=r_{0}$ gives

$$
\begin{aligned}
& -\chi^{2} \gamma_{1} J_{1}\left(\chi r_{0}\right)+\frac{a \kappa_{2}^{2} J_{1}\left(\kappa_{2} r_{0}\right)}{\kappa_{2}^{2}-\chi^{2}}+\frac{b \kappa_{1}^{2} J_{1}\left(\kappa_{1} r_{0}\right)}{\kappa_{1}^{2}-\chi^{2}}= \\
& \delta_{1} K_{1}\left(r_{0} / \rho_{\mathrm{i}}\right)+\frac{p \lambda_{2}^{2} K_{1}\left(\lambda_{2} r_{0}\right)}{\lambda_{2}^{2}-1 / \rho_{\mathrm{i}}^{2}}+\frac{q \lambda_{1}^{2} K_{1}\left(\lambda_{1} r_{0}\right)}{\lambda_{1}^{2}-1 / \rho_{\mathrm{i}}^{2}} .
\end{aligned}
$$

The continuity of the functions $D\left(\phi_{1}-B_{0} u_{y} x\right)$ and $G\left(\phi_{1}+\left(v_{0}-\right.\right.$ $\left.\left.u_{y}\right) B_{0} x-n_{1} \kappa T_{\mathrm{e}} /\left(e n_{0}\right)\right)$ is already satisfied by the previous continuity conditions. In order to make contour plots of the solutions, some of the constants and parameters can be chosen freely. A sketch of the solution is given in Figs. 5 and 6. Clearly, the solution is a double vortex, a structure that is spatially localized in the perpendicular direction and very elongated in the parallel direction. It consists of one positive and one negative vortex that are coupled and move together through the plasma. In Fig. 5 the magnetic field lines are perpendicular to the plane of the plot. The electric field is oriented in the direction perpendicular to the contour lines, with the strongest gradient around the separatrix denoted by the value 0 . Hence, due to the $\boldsymbol{E} \times \boldsymbol{B}$-drift, this is the region where very strong flux bursts of plasma particles are expected to occur along the separatrix.

Such a situation has been recently experimentally observed (Fredriksen et al. 2003). In fact, the same holds for the direction along the magnetic field lines because the structure is slightly slanted with respect to the $z$-axis, implying an electric field component along the magnetic field lines. The magnitude of this component is much smaller compared to that of the component in the perpendicular direction, but it acts on much larger scales and, therefore, can accelerate plasma particles. In view of the fact that the dipole consists of two spatially separated parts, such parallel fluxes appear in both its parts. Note also that these potential coherent structures are accompanied by two density vortices, represented by Eqs. (36) and (37), one representing a condensation and the other one representing a rarefaction of the density, like the structure presented in Fig. 6. 


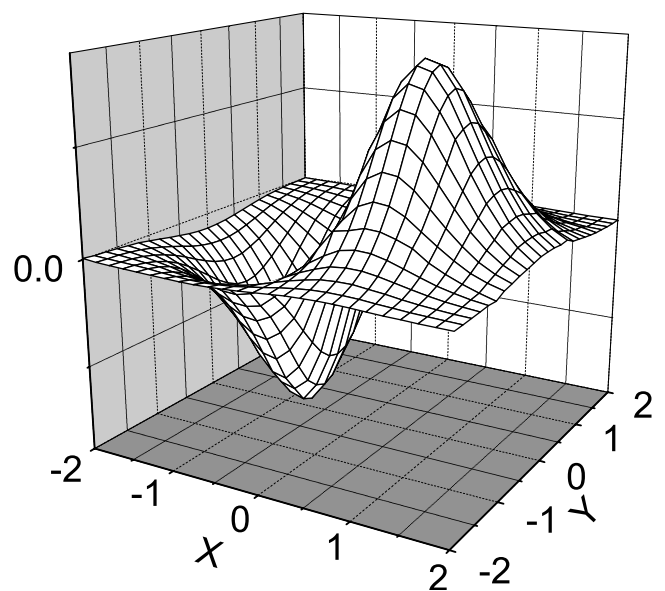

Fig. 6. The double vortex (in arbitrary units) as a sketch of the solution of Eq. (32).

Structures presented here may contribute to the formation of chromospheric spicules and coronal plumes. According to some models (Haerendel 1992), spicules and even coronal plumes may appear due to weakly damped (due to ion-neutral collisions) Alfvén waves. In such a scenario, Alfvén waves in combination with collisions result in a drag force which overpowers the gravitational force, and lifts cool and dense gas into higher layers. The model predicts the characteristic damping length $\lambda_{\text {damp }} \sim 1 / \omega^{2}>10^{4} \mathrm{~km}$ and frequencies $<0.35 \mathrm{~Hz}$; however, it requires a source capable of exciting the waves up to large amplitudes. The vortex as described here represents a possible natural self-organization of unstable and highly nonlinear perturbations with the source in the perpendicular electron drift.

\section{Summary and conclusions}

The dispersion relation has been derived for the coupled electron drift-driven and dispersive (kinetic) Alfvén modes in a strongly collisional plasma with an electron drift perpendicular to the magnetic field lines. For typical plasma parameter values of the solar photosphere, it has been shown that without the electron drift, the real part of the kinetic Alfvén wave frequency is practically zero, due to a strong collisional damping. In the presence of the electron drift, the Alfvén mode has a finite frequency, yet it remains damped. However, the drift-driven, Farley-Buneman type mode, is shown to be unstable when the electron drift velocity exceeds a certain threshold, as can be seen in Fig. 1.

The strong coupling between an instability (the drift-driven mode) and a strongly damped wave (the kinetic Alfvén mode) may have some potential for the problem of the heating and acceleration of solar atmospheric plasma. As a matter of fact, our results show that the instability is always present and, at the same time, always coupled to the heavily damped wave. As a result, the KAW will always be excited indirectly, viz. due to the neutral flow across the magnetic field lines. However, due to the strong damping, this wave energy will be efficiently converted into kinetic or thermal energy and thus accelerate or heat the ambient plasma. This qualitative picture should be investigated in more

detail, and it should be quantified to what extent this mechanism may contribute to the heating and acceleration of the solar atmospheric plasma. This, however, is not the concern of the present paper.

At spatial scales that far exceed the mean free path of plasma particles, a nonlinear analysis is performed showing the existence of traveling double vortices, which appear as particular solutions of the given set of three nonlinear equations for the scalar and vector potentials $\phi_{1}, A_{z 1}$, and the number density $n_{1}$. The nonlinearity studied here is of the vector-product type, and it appears through the convective derivatives in the momentum equations. It results in coherent structures for the three quantities that are highly elongated along the magnetic field lines and localized in the perpendicular direction. The double vortex consists of two parts with opposite rotations inside of them. The given profile of the electrostatic potential implies an electric field that has a component in the parallel direction and can consequently result in an acceleration of plasma particles. On the other hand, the isolines of the vector potential coincide with the perturbed (perpendicular) magnetic field lines, so that the total magnetic field lines within the vortex become twisted.

We stress that the model used here and introduced by Sen $\&$ White (1972), is in fact applicable to those solar magnetic structures that resemble bounded plasma configurations under laboratory conditions. Recent experimental investigations (Fredriksen et al. 2003) clearly show the formation of traveling double vortices in these bounded plasmas, where an opposite rotation inside each of the two vortex parts is clearly measured. Accompanied by such a dipole are strong bursts of plasma particles in the perpendicular direction, located in the area around the separatrix between the two parts. Consequently, the signatures in the solar plasma of the double vortices presented in the present work should be strong fluxes of plasma particles both in the perpendicular and parallel directions, with opposite motions of electrons and ions.

Acknowledgements. These results were obtained in the framework of the projects G.0451.05 (FWO-Vlaanderen), C90203 (Prodex), and GOA/2004/01 and OT/02/57 (K.U.Leuven).

\section{References}

Chen, F. F. 1984, Introduction to Plasma Physics and Controled Fusion (New York, London: Plenum Press), 351

Fredriksen, Å., Riccardi, C., \& Pécseli, H. 2003, Plasma Phys. Control. Fusion, 45,721

Giaretta, D. L. 1979, A\&A, 75, 237

Haerendel, G. 1992, Nature, 360, 241

Ichimaru, S. 1980, Basic pricinples of plasma physics (Reading, Massachusetts: The Benjamin/Cummings Pub.), 144

Khodachenko, M. L., \& Zaitsev, V. V. 2002, Ap\&SS, 279, 389

Larichev, V. D., \& Reznik, G. M. 1976, Dokl. Akad. Nauk SSSR, 231, 1077

Mitchner, M., \& Kruger, C. H. 1973, Partially Ionized Gasses (New York: Wiley), 413

Petrović, D., Vranjes, J., \& Poedts, S. 2005, Phys. Plasmas, 12, 112103

Priest, E. R. 1987, Solar Magnetohydrodynamics (Dordrecht/Boston/Lancaster: D. Reidel Publishing Company), 79

Sen, H. K., \& White, M. L. 1972, Sol. Phys., 23, 146

Spitzer, L. 1962, Physics of Fully Ionized Gasses (New York, London: Interscience), 146

Vernazza, J. E., Avrett, E. H., \& Loeser, R. 1981, ApJS, 45, 635

Vranjes, J. 1999, A\&A, 351, 1190

Weiland, J. 2000, Collective Modes in Inhomogeneous Plasmas (Bristol: Institute of Physics Pub.) 